\title{
TRATAMIENTO CON SILDENAFILO DEL SÍNDROME DE GLANDE FRIO TRAS IMPLANTE DE PRÓTESIS HIDRÁULICA DE PENE
}

\author{
E. LLEDÓ GARCIA, I. MONCADA IRIBARREN, J. JARA RASCÓN, \\ C. CARRERA PUERTA*, F. GONZÁLEZ-CHAMORRO*, C. LLORENTE ABARCA*, \\ C. HERNÁNDEZ FERNÁNDEZ
}

Servicio de Urología. Hospital General Universitario Gregorio Marañón. Madrid. *Servicio de Urología. Fundación Hospital Alcorcón. Alcorcón (Madrid).

Actas Urol Esp. 28 (6): 443-446, 2004

\section{RESUMEN}

TRATAMIENTO CON SILDENAFILO DEL SÍNDROME DE GLANDE FRIO TRAS IMPLANTE DE PRÓTESIS HIDRÁULICA DE PENE

OBJETIVO: Evaluar el efecto de sildenafilo en la respuesta peneana glandular durante la relación sexual (RS) en pacientes con prótesis de pene que muestran síntomas sugerentes de síndrome de glande frío (SGF).

MATERIAL Y MÉTODO: Evaluamos catorce pacientes a quienes se había implantado una prótesis de pene (PP) hidráulica de tres piezas. A pesar de un funcionamiento normal del dispositivo, todos los pacientes se quejaban de diversos grados de insatisfacción durante la RS debido a falta de incremento de volumen en glande. Se recomendó a los pacientes la ingestión de una dosis de $100 \mathrm{mg}$ de sildenafilo unos 45 minutos antes de activar la $\mathrm{PP}$ e iniciar la RS. Todos ellos siguieron este esquema al menos en tres ocasiones. Respondieron las cuestiones 7, 8, 13 y 14 del Índice Internacional de Función Eréctil (IIEF) con/sin dosis previa de sildenafilo.

RESULTADOS: Doce de los catorce pacientes $(85,7 \%)$ indicaron una RS más placentera tras ingerir sildenafilo. Este hecho fue relacionado especialmente con un incremento en la sensibilidad y volumen del glande. Las puntuaciones obtenidas en las cuestiones del IIEF mostraron un incremento significativo al tomar sildenafilo. Las parejas también referían una RS más confortable que relacionaron con una PV menos dolorosa. No se objetivó morbilidad relacionable con sildenafilo.

CONCLUSIONES: Sildenafilo puede ser utilizado para mejorar los resultados funcionales de las PP en pacientes con síndrome de glande frío y parece incrementar la satisfacción durante la RS.

PALABRAS CLAVE: Disfunción eréctil. Prótesis de pene. Sildenafilo. Síndrome de glande frío.

\footnotetext{
ABSTRACT

TREATMENT WITH SILDENAFIL OF COLD GLANDS SÍNDROME AFTER INFLATABLE PENILE PROSTHESIS

OBJECTIVE: To evaluate the effect of sildenafil on the response of glans penis during sexual intercourse in patients with penile prosthesis who show cold glans syndrome symptoms.

MATERIAL AND METHODS: Fourteen patients who had undergone three-piece inflatable penile prosthesis implantation were evaluated. In spite of the normal functioning of the device all of them complained of a degree of dissatisfaction during SI due to a lack of engorgement in the glans penis. The patients were advised to have a dose of $100 \mathrm{mg}$ VO sildenafil about 45 minutes before activating the PP and starting SI. All of them followed this scheme at least three times. They ticked questions 7, 8, 13 and 14 of the International Index of Erectile Function (IIEF) questionnaire with/without having had sildenafil.

RESULTS: Twelve out of thirteen patients (85,7\%) indicated a more pleasant SI on sildenafil. It was related to an increase in penile glans engorgement and sensitivity. The scores obtained in the IIEF questions showed a significant increase on taking sildenafil. The partners also talked of a more comfortable SI that they related to a less painful penetration. Sildenafil-related morbidity was not found.

CONCLUSIONS: Sildenafil could be used to improve functional results of the penile prosthesis in those patients with CGS and seems to increase satisfaction during SI.

KEY WORDS: Erectile dysfunction. Penile prosthesis. Sildenafil. Cold glans syndrome.
} 
A unque se han incorporado fármacos orales eficaces y seguros para el tratamiento de la disfunción eréctil (DE) el implante de prótesis de pene sigue siendo un tratamiento altamente satisfactorio para numerosos pacientes. Esta técnica ofrece excelentes resultados en términos funcionales y en cuanto a satisfacción de la pareja. El número de complicaciones es bajo cuando la intervención es realizada de forma sistematizada y rápida por cirujanos experimentados. Estudios recientes muestran porcentajes de satisfacción superiores al $85 \%^{1}$. La morbilidad del procedimiento puede ser intraoperatoria (lesiones uretrales o del septo intercavernoso, perforación crural) ${ }^{2}$. La aparición de infecciones post-operatorias o complicaciones mecánicas puede obligar al cirujano a re-intervenciones con el fin de reparar o, incluso de extraer los elementos de la prótesis. Más de un $17 \%$ de casos van a mostrar problemas funcionales a largo plazo. La insatisfacción del paciente a pesar de un buen funcionamiento del dispositivo puede también ser considerada una complicación. Por ejemplo, algunos pacientes refieren una pobre sensación de incremento de volumen y/o temperatura en el glande durante la RS. Ello hecho puede limitar la utilización satisfactoria de la prótesis. Este cuadro ha sido definido como "síndrome de glande frío" $(\mathrm{SGF})^{3}$.

El tratamiento de este problema no ha sido aún completamente establecido. La inyección intracavernosa de prostaglandina E1 está obviamente contraindicada en los pacientes con prótesis de pene. Se ha referido recientemente en la literatura la utilización eficaz de prostaglandina intrauretral (MUSE) con resultados alentadores $^{3,4}$. Sin embargo, la administración a medio y largo plazo de este producto implica una manipulación uretral periódica con el consiguiente riesgo de laceración mucosa e infecciones periuretrales secundarias. Por otro lado, esta forma de presentación no ha sido comercializada en España.

No hemos encontrado referencias previas en la literatura en relación a la utilidad de sildenafilo para el tratamiento del SGF. Presentamos un trabajo prospectivo con el objetivo de evaluar la eficacia de este fármaco en cuanto a mejorar la sensación de incremento de volumen y temperatura glandular así como el nivel de satisfacción obtenido en la RS en pacientes con prótesis peneana funcionante que refieren sintomas sugerentes de SGF.

\section{MATERIAL Y MÉTODO}

Evaluamos catorce pacientes a quienes se había implantado una prótesis hidráulica de tres componentes. Todos los pacientes referían a pesar de conseguir una adecuada rigidez al activar el dispositivo una carencia subjetiva de incremento de volumen y temperatura en el glande peneano durante la RS. Se indicó a todos ellos completar el siguiente esquema terapéutico:

- Período 1: duración de 4 semanas; los pacientes mantenían RS en su forma habitual.

- Período 2: duración de 4 semanas; los pacientes recibian una dosis de $100 \mathrm{mg}$ de sildenafilo unos 45 minutos antes de iniciar la RS.

En todos los casos se completaron ambos periodos de forma consecutiva y los pacientes realizaron RS con penetración vaginal (PV) al menos en tres ocasiones por período. Se solicitó la cumplimentación de las cuestiones $7,8,13$ y 14 del Índice Internacional de Función Eréctil (IIEF) después de cada uno de los dos períodos (Tabla 1).

\section{RESULTADOS}

Doce de catorce pacientes $(85,7 \%)$ refirieron una RS más satisfactoria al tomar sildenafilo. Este grupo relacionó este hecho con una percepción subjetiva de mayor incremento de volumen, temperatura y sensibilidad en el glande durante

Tabla 1. Cuestiones 7, 8, 13 y 14 del Índice Internacional de Función Eréctil (IIEF)

7. Durante las últimas 4 semanas, cuando intentó el acto sexual (penetración vaginal), ¿con qué frecuencia resultó satisfactorio para usted?

8. Durante las últimas 4 semanas, ¿cuánto ha disfrutado del acto sexual (penetración vaginal)?

13. Durante las últimas 4 semanas, ¿cuál ha sido el grado de satisfacción con su vida sexual en general?

14. Durante las últimas 4 semanas, ¿cuál ha sido el grado de satisfacción con la relación sexual con su pareja? 
la PV, la cual explicaron, además, como más fácil. Las parejas de estos pacientes también reconocieron la PV como más cómoda y menos dolorosa. Las puntuaciones obtenidas en las cuestiones seleccionadas del IIEF mostraron una variación significativa (Tabla 2) al comparar los resultados sin/con ingestión previa de la medicación. No observamos morbilidad significativa relacionada con la toma de sildenafilo.

Tabla 2. Puntuaciones obtenidas por los pacientes en las cuestiones del IIEF $\sin /$ con dosis previa de sildenafilo

\begin{tabular}{cccc}
\hline $\begin{array}{c}\text { Cuestión } \\
\text { IIEF }\end{array}$ & $\begin{array}{c}\text { RS sin sildenafilo } \\
\text { previo }\end{array}$ & $\begin{array}{c}\text { RS con sildenafilo } \\
\text { previo }\end{array}$ & p \\
\hline $7+8$ & 5 & 9 & $<0,05$ \\
$13+14$ & 7 & 10 & $<0,05$ \\
\hline
\end{tabular}

\section{DISCUSIÓN}

Un adecuado incremento de volumen del glande peneano durante la RS es importante para conseguir que la PV sea más funcional y confortable tanto para el varón como para su pareja. En los pacientes con $\mathrm{DE}$, la carencia de rigidez peneana axial efectiva no se produce sólo a expensas de los cuerpos cavernosos, sino también del tejido eréctil esponjoso periuretral y glandular. Algunos pacientes con PP funcionantes refieren grados variables de insatisfacción durante la penetración que relacionan con un incremento insuficiente en el volumen y temperatura del glande (síndrome de glande frío, SGF). Este glande "blando" puede dificultar la PV y hacer ésta molesta también para la pareja, debido a la pérdida de efecto amortiguador del tejido esponjoso glandular repleccionado sobre la parte más distal de los cilindros protésicos. Teniendo en cuenta las características histológicas del tejido esponjoso eréctil glandular, estos pacientes pueden beneficiarse de una estimulación farmacológica vascular.

Los cuerpos cavernosos son dos estructuras cilíndricas ubicadas dorsalmente en el pene. Desde el punto de vista geométrico, son elementos tubulares interconectados a través de una línea media multiperforada y pueden, desde este punto de vista, considerarse un solo espacio vascular ${ }^{5}$.
Cada cuerpo cavernoso está envuelto en una gruesa vaina fibrosa, la túnica albugínea. Dos estratos pueden distinguirse en esta estructura: una capa externa longitudinal y otra interna circular. Ventralmente, la túnica albuginea forma una especie de "techo" para el cuerpo esponjoso. El tejido eréctil está formado por múltiples espacios lacunares, interconectados y tapizados por un revestimiento celular endotelial. Las trabéculas constituyen las paredes de estos espacios y están formadas por músculo liso (45\%) y una trama fibroelástica compuesta principalmente por colágeno ${ }^{6}$.

La uretra está completamente envuelta por el tejido esponjoso a lo largo de su recorrido, delimitado entre los dos cuerpos cavernosos y en la porción pendular del pene. Proximalmente va a formar el bulbo, que está unido a la porción inferior del diafragma urogenital. Distalmente, el cuerpo esponjoso se expande formando el glande del pene. El tejido esponjoso está constituido por espacios sinusoidales de mayor tamaño que los observados en los cuerpos cavernosos y con menos cantidad de tejido muscular liso. Su túnica albugínea es más fina. La capacidad erectógena del tejido esponjoso es también menos importante en comparación con el tejido cavernoso. Sin embargo, el incremento de volumen del glande es parte funcional esencial de la erección. Este aspecto concreto dependerá especialmente del incremento de flujo sanguíneo a través de la arteria dorsal del pene.

Según Carlson ${ }^{3}$, la administración intrauretral de alprostadilo $\left(\mathrm{MUSE}^{\mathrm{R}}\right)$ resulta en un mayor incremento de volumen glandular durante la RS y PV en los pacientes con PP funcionante afectados por SGF. Chew ${ }^{4}$ refiere conclusiones similares. Ambos autores atribuyen sus resultados a la presencia de conexiones entre las venas emisarias que provienen de los cuerpos cavernosos y sus homólogas circunflejas que provienen del cuerpo esponjoso. Este hecho anatómico permitiría el paso de alprostadilo al compartimento esponjoso después de su absorción a través de la mucosa uretral.

Teóricamente la administración de sildenafilo oral puede actuar sobre el tejido erectogénico intacto, también en aquellos pacientes con cirugías previas sobre el mismo. La anatomía peneana es 
obviamente alterada después del implante de una prótesis. El tejido cavernoso eréctil es destruido por las maniobras quirúrgicas y sustituido por cilindros, sólo con un remanente que yacerá comprimido en la porción interna más periférica de los cuerpos cavernosos. La estructura del cuerpo esponjoso periuretral y glandular, sin embargo, permanece intacto junto con sus propiedades erectógenas. Sildenafilo puede potencialmente mantener su efecto bioquímico en estos tejidos, lo que depende específicamente de inhibir la acción de la PDE5.

Nuestros pacientes acudieron a la consulta con la queja de PV insatisfactorias tanto para ellos como para sus parejas, a pesar de un buen funcionamiento de la prótesis. Ellos fueron instruidos para completar consecutivamente dos periodos de actividad sexual, con la diferencia de que durante el segundo periodo de cuatro semanas deberían ingerir una dosis de $100 \mathrm{mg}$ de sildenafilo antes de cada intento de RS. Los pacientes podían activar la prótesis de la forma que habitualmente utilizaran y sólo se les aconsejaba el incluir en su RS una fase los más intensa posible de estimulación erótica antes de intentar la PV en todos los casos. Después de seguir estas instrucciones los pacientes mostraron un incremento significativo en la puntuación obtenida en las cuestiones 7-8-13-14 del IIEF en un 85\% de los casos. Esto parece demostrar un significativo aumento en el nivel de satisfacción tanto sexual global como durante la PV en los pacientes con sintomas sugerentes de SGF, al tomar sildenafilo antes de la RS.

Los pacientes con incrementos significativos en la puntuación de las cuestiones del IIEF habían mostrado respuestas parciales a la inyección intracavernosa y/o a la administración oral de sildenafilo antes de indicar el implante de la prótesis de pene. Sin embargo, los dos pacientes en nuestra serie con SGF y sin incrementos significativos en la puntuación del IIEF habían mostrado ausencia completa de respuesta a farmacoterapia local/sistémica antes del implante de la prótesis. Parece importante el tener en cuenta que todos los pacientes respondedores relacionaron directamente su mayor confortabilidad peneana durante la PV con un incremento en el volumen y sensibilidad del glande. Tanto los propios pacientes como sus parejas hicieron hincapié en la mayor facilidad tras la toma del fármaco para conseguir la penetración que resultó claramente menos dolorosa para las parejas.

\section{CONCLUSIONES}

Sildenafilo puede utilizarse para mejorar los resultados funcionales de las prótesis de pene en aquellos pacientes con síntomas sugerentes de SGF. El fármaco parece incrementar el nivel de satisfacción sexual global y durante la PV tanto en los propios pacientes, como, en consecuencia, en sus parejas.

\section{REFERENCIAS}

1. CARSON CC, MULCAHY JJ, GOVIER FE.: Efficacy and safety of outcomes of the AMS $700 \mathrm{CX}$ inflatable penile prosthesis: results of a long-term, multicenter study. J Urol 1999 suppl., 161: 259, abstract 999.

2. WILSON SK.: Management of penile implant complications. In Mulcahy JJ.: Topics in clinical Urology. Diagnosis and management of male sexual dysfunction. Williams \& Wilkins 1997; 14: 231-253.

3. BENEVIDES MD, CARSON CC.: Intraurethral application of alprostadil in patients with failed inflatable penile prosthesis. J Urol 2000; 163: 785.

4. CHEW KK, STUCKEY BG.: Use of transurethral alprostadil (MUSE) (prostaglandin E1) for glans tumescence in a patient with penile prosthesis. Int $J$ Impot Res 2000; 12 (3): 195-196.

5. GOLDSTEIN AMB, MEEHAN JP, MORROW JW.: The fibrous skeleton of the corpora cavernosa and its probable function in penile erection. $\mathrm{Br} J$ Urol 1985; 57: 574.

6. GOLDSTEIN AMB, PADMA-NATHAN H.: The microarchitecture of the intracavernosal smooth muscle and the cavernosal fibrous skeleton. $J$ Urol 1990; 144: 1144.

Dr. E. Lledó García

Servicio de Urología

Hosp. Gral. Univ. Gregorio Marañón

C/ Doctor Esquerdo, 46

28007 Madrid

(Trabajo recibido el 17 noviembre de 2003) 